\title{
Subclinical Atrial Fibrillation and the Risk of Recurrent Ischemic Stroke
}

\author{
Rolf Wachter ${ }^{1,2}$ Ben Freedman 3,4 \\ ${ }^{1}$ Clinic and Policlinic for Cardiology, University Hospital Leipzig, \\ Leipzig, Germany \\ ${ }^{2}$ German Cardiovascular Research Center, partner site Göttingen, \\ Göttingen, Germany \\ ${ }^{3}$ Heart Research Institute, Charles Perkins Centre, University of \\ Sydney, Camperdown, Sydney, NSW, Australia \\ ${ }^{4}$ Department of Cardiology, Concord Hospital, Hospital Road, \\ Concord, NSW, Australia
}

Thromb Haemost 2021;121:697-699.

In 2014, Hart et al introduced the clinical construct of "embolic stroke of unknown source" (ESUS). ${ }^{1}$ In brief, it describes a subgroup of stroke patients in whom, based on brain imaging, cardioembolism is highly suggestive to be the putative stroke mechanism. Anticoagulation should be a better choice for these patients than antiplatelet therapy and this concept was subsequently investigated in three randomized trials.

The NAVIGATE-ESUS trial randomized 7,213 patients with a recent ESUS to either rivaroxaban $15 \mathrm{mg}$ or aspirin $100 \mathrm{mg}^{2}$ The trial was stopped prematurely for futility and harm: the primary endpoint of stroke and systemic embolism was not different in both groups (5.1\%/year [rivaroxaban] vs. $4.8 \%$ / year [aspirin]), while major bleedings were nearly tripled with rivaroxaban (1.8 vs. $0.7 \% /$ year).

The RESPECT-ESUS trial assigned 5,390 patients with ESUS to either dabigatran ( 150 or $110 \mathrm{mg}$ twice daily based on age and kidney function) or aspirin. ${ }^{3}$ The primary endpoint of recurrent stroke was not different between the groups (4.1\%/year with dabigatran vs. $4.8 \% /$ year with aspirin). Nonmajor bleedings were also not different between the groups, but clinically relevant nonmajor bleeding occurred more often with dabigatran (1.6\%/year) than with aspirin (0.9\%/year).

A third trial called ATTICUS is smaller with a planned inclusion of only 500 patients and a brain imaging endpoint, ${ }^{4}$ but was stopped recently for futility (Sven Poli, personal communication).

So, does the concept of ESUS belong to the "dustbin of history" (R. Hart, quote)? Yes, in terms of generally using anticoagulation in all with ESUS. No, in terms of pointing to a need to further identify a subgroup of ESUS with cardioembolism that may benefit from anticoagulation. ${ }^{5}$

\section{received}

February 14, 2021

accepted after revision

February 15, 2021

published online

April 28, 2021

Address for correspondence Ben Freedman, MBBS, PhD, Heart Research Institute, Charles Perkins Centre, University of Sydney, Camperdown, Sydney, NSW 2006, Australia

(e-mail: ben.freedman@sydney.edu.au).

One group that without any doubt benefits from anticoagulation for stroke prevention is patients with atrial fibrillation (AF). But AF might escape routine diagnostics in stroke patients as it is often paroxysmal. ${ }^{5}$ The most sensitive method for AF detection is continuous monitoring by an implantable cardiac monitor. This type of device-detected AF is also called subclinical $\mathrm{AF}$ (SCAF) or atrial high rate episodes if found in implanted cardiac devices like pacemakers with an atrial lead. ${ }^{5}$ Recent evidence suggests that SCAF is a frequent phenomenon in patients with implanted cardiac devices, ${ }^{6}$ but also in patients with cardiovascular risk factors having continuous electrocardiogram monitoring by a subcutaneous implanted cardiac monitor. ${ }^{7-9}$ It is important to note that the risk of stroke is substantially lower than in patients with clinical AF, though structural changes of the left atrium have to be taken into account-a higher stroke risk in those with left atrial dilatation. ${ }^{10}$ The efficacy of oral anticoagulants in patients with SCAF is currently being investigated in two randomized trials: ARTESIA (NCT01938248) and NOAH-AFNET6 (NCT02618577). However, the percentage of patients with a history of stroke is rather low (e.g., 4.5\% in ARTESIA [Jeff Healey, personal communication]).

The role of SCAF in stroke patients is uncertain. ${ }^{11,12}$ In this issue of Thrombosis and Haemostasis, Kitsiou et al report the 3-year follow-up data of a prospective observational study in 123 patients with ESUS, ${ }^{13}$ which extends the initial findings published in 2017. ${ }^{14}$ They report a cumulative SCAF prevalence of $41 \%$ and a stroke recurrence rate of $23 \%$. The proportion of patients with SCAF is rather similar to studies in patients with cardiovascular risk factors or implanted devices, but the stroke recurrence rate is six to 10 times higher (-Fig 1). Therefore, the analysis of patients who

(c) 2021. Thieme. All rights reserved. Georg Thieme Verlag KG,

Rüdigerstraße 14,

70469 Stuttgart, Germany
DOI https://doi.org/ 10.1055/s-0041-1726403. ISSN 0340-6245. 


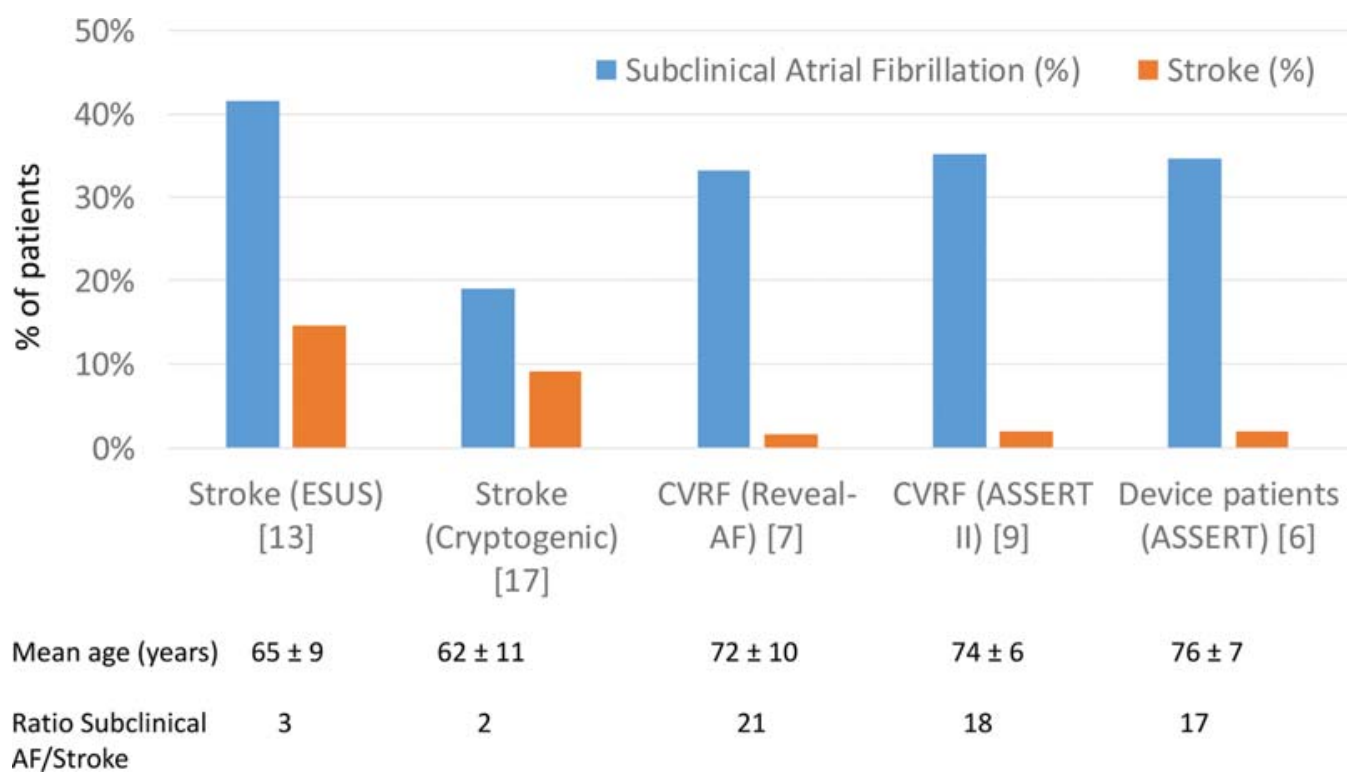

Fig. 1 Ratio of rate of subclinical AF and rate of stroke in implanted monitors and cardiac devices. Percentage of patients with subclinical atrial fibrillation detected by continuous monitoring and stroke rate in the two stroke studies (ESUS patients in the current study, ${ }^{13}$ cryptogenic stroke patients in the CRYSTAL-AF study ${ }^{17}$ ), in two studies of patients with cardiovascular risk factors (CVRF) (Reveal-AF, ${ }^{7}$ ASSERT- $2^{9}$ ) and in one study in patients with implanted devices (ASSERT ${ }^{6}$ ). The mean age was higher in the nonstroke studies, but the stroke risk substantially lower.

experience SCAF and a recurrent stroke is of crucial importance. In five patients, SCAF preceded the stroke while in the other four, recurrent stroke occurred before the SCAF. Two patients with SCAF were insufficiently anticoagulated when the stroke occurred.

Most evidence regarding the risk for stroke comes from the ASSERT trial. ${ }^{6}$ While the overall risk of stroke was 2.5 higher in patients with SCAF, there was no clear temporal relationship between SCAF and the stroke event, i. e., only $8 \%$ of patients had SCAF within 30 days before stroke. ${ }^{15}$ This seems similar to patients after ESUS as found in the current study ${ }^{13}$ : none of the patients had SCAF in the 30 days before the stroke. Another subanalysis from the ASSERT trial informed that SCAF increases the risk only if one episode exceeds 24 hours. ${ }^{16} \mathrm{~A}$ total of $78 \%$ of the recurrent stroke events were again ESUS.

Some limitations have to be acknowledged. The duration of the longest SCAF episode during follow-up was not collected in the study because the implantable cardiac monitor was explanted after the first detection of SCAF. The study is single-center study and all patients with SCAF received anticoagulation, which limits conclusions to be made on the natural history of SCAF post-ESUS.

So, what do we learn from the current study? SCAF is a frequent finding after ESUS and the stroke recurrence risk in these patients is very high ( $23 \%$ in 3 years). There is no temporal relationship between SCAF and stroke though the impact of longer duration of SCAF on stroke risk was not assessed. This evidence suggests that an atrial myopathy may cause both the stroke and the SCAF. ${ }^{12}$ Future studies with implantable loop recorders should continue monitoring after the first documentation of SCAF and ideally randomize these patients to anticoagulation versus antiplatelet therapy. And other studies such as the ongoing ARCADIA study
(NCT03192215), which is randomizing patients with prior ESUS and evidence of atrial cardiomyopathy to apixaban or aspirin, should help determine whether evidence of atrial myopathy might be used as a guide to the need for anticoagulation rather than intensively looking for SCAF.

Conflict of Interest

None declared.

\section{References}

1 Hart RG, Diener HC, Coutts SB, et al; Cryptogenic Stroke/ESUS International Working Group. Embolic strokes of undetermined source: the case for a new clinical construct. Lancet Neurol 2014; 13(04):429-438

2 Hart RG, Sharma M, Mundl H, et al; NAVIGATE ESUS Investigators. Rivaroxaban for stroke prevention after embolic stroke of undetermined source. N Engl J Med 2018;378(23):2191-2201

3 Diener HC, Sacco RL, Easton JD, et al; RE-SPECT ESUS Steering Committee and Investigators. Dabigatran for prevention of stroke after embolic stroke of undetermined source. N Engl J Med 2019; 380(20):1906-1917

4 Geisler T, Poli S, Meisner C, et al. Apixaban for treatment of embolic stroke of undetermined source (ATTICUS randomized trial): rationale and study design. Int J Stroke 2017;12(09): 985-990

5 Schnabel RB, Haeusler KG, Healey JS, et al. Searching for atrial fibrillation poststroke: a white paper of the AF-SCREEN International Collaboration. Circulation 2019;140(22):1834-1850

6 Healey JS, Connolly SJ, Gold MR, et al; ASSERT Investigators. Subclinical atrial fibrillation and the risk of stroke. N Engl J Med 2012;366(02):120-129

7 Reiffel JA, Verma A, Kowey PR, et al; REVEAL AF Investigators. Incidence of previously undiagnosed atrial fibrillation using insertable cardiac monitors in a high-risk population: the REVEAL AF study. JAMA Cardiol 2017;2(10):1120-1127

8 Nasir JM, Pomeroy W, Marler A, et al. Predicting determinants of atrial fibrillation or flutter for therapy elucidation in patients at 
risk for thromboembolic events (PREDATE AF) study. Heart Rhythm 2017;14(07):955-961

9 Healey JS, Alings M, Ha A, et al; ASSERT-II Investigators. Subclinical atrial fibrillation in older patients. Circulation 2017;136(14): 1276-1283

10 Potpara TS, Lip GYH, Blomstrom-Lundqvist C, et al. The 4S-AF scheme (stroke risk; symptoms; severity of burden; substrate): a novel approach to in-depth characterization (rather than classification) of atrial fibrillation. Thromb Haemost 2020;121(03):270-278

11 Wachter R, Freedman B. The role of atrial fibrillation in patients with an embolic stroke of unknown source (ESUS). Thromb Haemost 2017;117(10):1833-1835

12 Freedman B, Kamel H, van Gelder IC, Schnabel RB. Atrial fibrillation: villain or bystander in vascular brain injury. Eur Heart J Suppl 2020;22:M51-M59

13 Kitsiou A, Rogalewski A, Kalyani M, et al. Atrial fibrillation in patients with embolic stroke of undetermined source during 3 years of prolonged monitoring with an implantable loop recorder. Thromb Haemost 2021;121(05): 826-833

14 Israel C, Kitsiou A, Kalyani M, et al. Detection of atrial fibrillation in patients with embolic stroke of undetermined source by prolonged monitoring with implantable loop recorders. Thromb Haemost 2017;117:1962-1969

15 Brambatti M, Connolly SJ, Gold MR, et al; ASSERT Investigators. Temporal relationship between subclinical atrial fibrillation and embolic events. Circulation 2014;129(21): 2094-2099

16 Van Gelder IC, Healey JS, Crijns HJGM, et al. Duration of devicedetected subclinical atrial fibrillation and occurrence of stroke in ASSERT. Eur Heart J 2017;38(17):1339-1344

17 Sanna T, Diener HC, Passman RS, et al; CRYSTAL AF Investigators. Cryptogenic stroke and underlying atrial fibrillation. N Engl J Med 2014;370(26):2478-2486 